\title{
Is waist-to-height ratio the best predictive indicator of cardiovascular disease incidence in hypertensive adults? A cohort study
}

\section{Yingxian Sun ( $\nabla$ yxsun@cmu.edu.cn )}

China Medical University First Hospital

\section{Shu Zhang}

The First Hospital of China Medical University

\section{Xin Fu}

Shenyang Medical College

Zhi Du

China Medical University Hospital

\section{Xiaofan Guo}

China Medical University Hospital

\section{Guozhe Sun}

China Medical University Hospital

\section{Zhao Li}

China Medical University Hospital

\section{Ying Zhou}

China Medical University Hospital

Hongmei Yang

China Medical University Hospital

\section{Shasha Yu}

China Medical University Hospital

\section{Liqiang Zheng}

China Medical University Second Hospital: Shengjing Hospital of China Medical University

\section{Research}

Keywords: cardiovascular disease, hypertensive adults, WHtR

Posted Date: August 3rd, 2021

DOI: https://doi.org/10.21203/rs.3.rs-744910/v1 
License: (c) (i) This work is licensed under a Creative Commons Attribution 4.0 International License. Read Full License 


\section{Abstract \\ Background}

Cardiovascular disease (CVD) brings high mortality and economic burden to patients, especially in rural areas. Simple, low-cost abdominal adiposity measures may help identify individuals with increased CVD risk. It is unclear that which obesity indice is the best to predict CVD in hypertensive people.

\section{Methods}

NCRCHS is a prospective cohort study in a general population in Northeast China. The study examined the cardiovascular health from 2013-2015, and follow-up captured the CVD incidence in 2018. Baseline waist-to-height ratio (WHtR), body mass index (BMI), waist-to-hip(WHR)and waist circumference (WC) were calculated and analyzed in relation to the CVD incidence.

\section{Results}

A total of 4244 hypertensive adults without pre-existing CVD at baseline were included in this analysis (age 35-92 years; 2108 men). Over a median follow-up of 4.66 years, a total of 290 CVD cases (6.83\%) were documented during the follow-up. Baseline WHtR showed a significant positive association with CVD incidence, even after adjusting for age, sex, diabetes, drinking, smoking, SBP, DBP, Triglyceride, HDLC, LDL-C, and TC (Hazard Ratios per SD of WHtR ranging from 1.03 to 1.31, $p=0.017$ ). Reclassification and discrimination analyses indicated WHtR addition could improve the conventional model for predicting adverse outcomes within 4 years. Moreover, WHtR predicted the CVD incidence better than other obesity indices (BMI, WC, WHR).

\section{Conclusion}

These findings support a positive association between WHtR and CVD incidence in CVD-free hypertensive adults. WHtR may be useful in predicting CVD incidence in hypertensive adults.

\section{Background}

Cardiovascular disease (CVD), has becoming a public health challenge caused by an aging global population [1-3]. In China, CVD is the leading cause of death. It accounts for $45.01 \%$ of total deaths in rural areas in 2015, while the proportion of urban areas is relatively lower than rural areas. The number of CVD patients is on the rise and predicted to increase substantially over the next 10 years. In particular, the mortality of the CVD increased from 150/100,000 in 1990 to 298/100,000 in 2015 in rural areas in China [4]. 
Obesity, particularly abdominal, is a key risk factor of cardiovascular disease (CVD) [5-7]. Using anthropometric indices to categorize obesity such as body mass index (BMI), the waist-to-height ratio (WHtR), the waist-to hip ratio (WHR), and waist circumference (WC)[8] are the simplest and the most costeffective methods recommended in clinical practice and epidemiological research, especially in developing countries. Some studies demonstrated that WHtR was better for predicting CVD risk, but it is unclear in the hypertensive adults [9-10].

Hypertension (HTN) is regarded as a serious public health problem [11-13]. The prevalence of HTN has been increasing all over the world [14-16]. However, it is not known that which anthropometric indice is the best to predict CVD in people with hypertension. Thus, elucidation of the best predictive indicator of cardiovascular disease incidence in hypertension adults is of great significance.

In the present study, we aimed to determine the role of WHtR as a predictor of CVD incidence in the NCRCHS (the Northeast China Rural Cardiovascular Health Study) cohort of hypertensive adults without previous CVD, and compare its discriminating ability against other commonly anthropometric indices of central obesity (i.e., WC, WHR and BMI).

\section{Methods}

\section{Study population}

NCRCHS is a prospective cohort study in a general population in Northeast China. The methods of the study, including research design, personnel recruitment, and data collection, have been elaborated in previous publications [16-17]. Briefly, using multistage, randomly stratified, cluster-sampling, 11956 participants aged at least 35 years were recruited from rural areas of Liaoning province between January 2013 and August 2013. Subsequently, participants were invited to attend follow-up visits in 2015 and 2018, and 6017 hypertensive participants were consented and eligible for the follow-up study. A total of 5249 participants of hypertension completed at least one follow-up visit. In the current study, we excluded baseline history of coronary heart disease $(n=355)$ and stroke $(n=590)$, and missing physical indicators $(n=60)$. Eventually, data from 4244 participants were available for analysis. The Ethics Committee of the

First Hospital of China Medical University (Shenyang, China) approved the study. All participants provided written informed consent.

\section{Data collection}

Data was collected during a single clinic visit by cardiologists and trained nurses using a standard questionnaire by face-to-face interview. Before the survey was performed, we invited all eligible investigators to attend the organized training. The training contents included the purpose of this study, how to administer the questionnaire, the standard method of measurement, the importance of standardization, and the study procedures. A strict test was evaluated after this training, only those who scored perfectly on the test could become investigators. During data collection, our inspectors had further instructions and support. 
All participants were asked about the current status of smoking, drinking and the history of diseases. Base on the recommendations of the Working Group on Obesity in China, participants were stratified according to the BMI levels as underweight group (BMI $18.5 \mathrm{~kg} / \mathrm{m}^{2}$ ), normal weight group (18.5 $\mathrm{kg} / \mathrm{m}^{2} \leq$ BMI $\left.\otimes 24 \mathrm{~kg} / \mathrm{m}^{2}\right)$, overweight $\left(24 \mathrm{~kg} / \mathrm{m}^{2} \leq \mathrm{BMI} \otimes 30 \mathrm{~kg} / \mathrm{m}^{2}\right)$, or obesity $\left(\mathrm{BMI} \geq 30 \mathrm{~kg} / \mathrm{m}^{2}\right)$. WHtR was calculated as WC divided by height. According to the reports from Ashwell, we categorized WHtR as WHtR $\nabla 0.40,0.40 \leq \mathrm{WH} t \mathrm{t} \leq 0.50,0.50 \otimes \mathrm{WH} t \mathrm{R} \leq 0.60$, WHtR $\otimes 0.60$. The group with $0.40 \leq \mathrm{WHtR} \leq 0.50$ was used as the reference group [18-20].

According to American Heart Association protocol, blood pressure was measured three times at 2-min intervals after at least 5 min of rest using a standardized automatic electronic sphygmomanometer (HEM-907; Omron). The participants were advised to avoid caffeinated beverages and exercise for at least 30 min before the measurement. During the measurement, the participants were seated with the arm supported at the level of the heart. The mean of three blood pressure measures was calculated and used in all analyses. Hypertension was defined as a mean SBP at least $140 \mathrm{mmHg}$ and/or a mean DBP at least $90 \mathrm{mmHg}$, and/or use of antihypertensive medication in the previous 2 weeks [21-22]. Diabetes mellitus was defined as FBG at least $7.0 \mathrm{mmol} / \mathrm{I}$ and/or self-reported physician-confirmed diagnosis [23]. Fasting blood samples were collected in the morning after at least $10 \mathrm{~h}$ of fasting. Blood samples were obtained from an antecubital vein into BD Vacutainer tubes containing ethylenediaminetetraacetic acid. Serum was subsequently isolated from the whole blood, and all serum samples were frozen at $-80^{\circ} \mathrm{C}$ for testing at a central, certified laboratory. Triglycerides, total cholesterol, low-density lipoprotein cholesterol, highdensity lipoprotein cholesterol and other blood biochemical indexes were analyzed enzymatically using the an Olympus AU640 autoanalyzer (Olympus, Kobe, Japan). All blinded duplicate samples were used for these analyses.

\section{Judgment and definition of clinical outcomes}

For all participants, all available clinical information on possible diagnoses or mortality was collected, including data from medical records and death certificates. Subsequently, materials were independently reviewed and adjudicated on by an endpoint assessment committee. CVD was defined as stroke or Coronary heart disease (CHD). Stroke was defined as rapidly developing signs of focal or global cerebral function disturbance lasting more than $24 \mathrm{~h}$ (unless interrupted by surgery or death) with no apparent nonvascular causes, based on the WHO Multinational Monitoring of Trends and Determinants in CVD criteria [24]. CHD was defined as a diagnosis of angina requiring hospitalization, miocardial infarction (MI) requiring hospitalization, CHD-related mortality, or any revascularization procedure [25].

\section{Statistical analysis}

Descriptive statistics were calculated for all variables, including continuous variables (reported as means and SDs) and categorical variables (reported as frequencies and percentages). As appropriate, differences between categories were evaluated using the $t$ test, or the $x 2$ test. Kaplan-Meier method was used to calculate the cumulative incidence for adverse events, and log-rank test was used to compare 
differences. Besides, to evaluate the improvement in risk prediction for adverse outcomes by adding WHtR to the conventional model (including age, sex, current smoking, current drinking, SBP, DBP, TC, HDL$\mathrm{C}$, LDL-C, triglyceride, and diabetes), we calculated the net reclassification improvement (NRI) and integrated discrimination improvement (IDI) for CVD prediction models respectively (conventional model vs. conventional model+ WHtR). The calculation method is IDI=(Pnew, events-Pold, events)-(Pnew, nonevents-Pold, non-events). With the larger value of IDI, the new model has the better prediction ability.

SPSS software version 22.0 (SPSS Inc., Chicago, Illinois, USA) and statistical software packages $\mathrm{R}$ (http://www. R-project.org, The R Foundation) were used for statistical analyses. P values were considered to be statistically significant if less than 0.05 .

\section{Results}

1. Baseline characteristics of the study sample according to CVD incidence

In this study, there are 6017 hypertensive participants consented and eligible for the follow-up study. A total of 5249 participants of hypertension completed at least one follow-up visit. In the current study, we excluded baseline history of coronary heart disease $(n=355)$ and stroke $(n=590)$, and missing physical indicators $(n=60)$. Presents the baseline characteristics of participants according to the CVD incidence. 4244 participants ( 2108 men and 2136 women, mean age $56.26 \pm 10.15$ years) were included in this cohort study (Supplemental figure S1). During a median follow-up of 4.66 years, 290 participants (6.83\%) incident stroke or CHD (crude incidence rate, 14.66 incident stroke or CHD per 1000 person-years).

The group of participants who developed CVD during the study follow-up consisted mainly of older men and exhibited higher anthropometric indices/ratios of total and central obesity (BMI, WC, WHR, WHtR), compared to those who remained CVD-free. Furthermore, this group had higher baseline DBP, SBP levels and lipids (TC and LDL-C) (all p-values < 0.05; Table 1). The mean WHtR value at baseline was $2 \%$ higher in the group of participants who developed a CVD event than in those who remained CVD-free $(p<0.05$, Table 1).

Table 1. Baseline characteristics of the study sample. 


\begin{tabular}{|c|c|c|c|c|}
\hline Variable & & $\begin{array}{l}\text { Without CVD (N = } \\
\text { 3954) }\end{array}$ & $\begin{array}{l}\text { With CVD (N = } \\
290)\end{array}$ & $\begin{array}{l}\mathrm{P} \\
\text { value }\end{array}$ \\
\hline Age (years) & & $55.85( \pm 10.10)$ & $61.85( \pm 9.26)$ & $<0.001$ \\
\hline Male [n (\%)] & & $1945(50.8 \%)$ & 163(56.2\%区 & 0.021 \\
\hline Current smoking [n (\%)] & & 1406(35.6\%区 & 118(40.8\%区 & 0.090 \\
\hline Current drinking [n (\%)] & & $1065(26.9 \% \square$ & $87(30.0 \% \square$ & 0.287 \\
\hline Body mass index $\left(\mathrm{kg} / \mathrm{m}^{2}\right)$ & & $25.55( \pm 3.58)$ & $25.79( \pm 3.54)$ & 0.268 \\
\hline \multirow{2}{*}{$\begin{array}{l}\text { Waist circumference }(\mathrm{cm}) \\
\text { ratio }\end{array}$} & \multirow[t]{3}{*}{ Waist-to-hip } & $84.39( \pm 9.52)$ & $85.80( \pm 10.49)$ & 0.016 \\
\hline & & $0.87( \pm 0.07 \rrbracket$ & $0.89( \pm 0.10 \rrbracket$ & $<0.001$ \\
\hline Waist-to-height ratio & & $0.53( \pm 0.06 \rrbracket$ & $0.54( \pm 0.06 \rrbracket$ & 0.001 \\
\hline $\mathrm{SBP}(\mathrm{mmHg})$ & & $157.90( \pm 18.18)$ & 168. $47( \pm 23.21)$ & $<0.001$ \\
\hline $\mathrm{DBP}(\mathrm{mmHg})$ & & $88.69( \pm 10.78)$ & $91.47( \pm 14.12)$ & 0.001 \\
\hline $\mathrm{TC}(\mathrm{mmol} / \mathrm{L})$ & & $5.40( \pm 1.09)$ & $5.55( \pm 1.11)$ & 0.023 \\
\hline LDL-C (mmol/L) & & $3.08( \pm 0.85)$ & $3.22( \pm 0.91)$ & 0.017 \\
\hline $\mathrm{HDL}-\mathrm{C}(\mathrm{mmol} / \mathrm{L})$ & & $1.45( \pm 0.41)$ & $1.45( \pm 0.45)$ & 0.909 \\
\hline Triglyceride (mmol/L) & & $1.76( \pm 1.65)$ & $1.77( \pm 1.53)$ & 0.978 \\
\hline Diabetes [n (\%)] & & 192ه4.9\%》 & 19囚6.6\%】 & 0.253 \\
\hline
\end{tabular}

Abbreviations: SBP, systolic blood pressure; DBP, diastolic blood pressure; TC, total cholesterol; LDL-C, low-density lipoprotein cholesterol; HDL-C, high-density lipoprotein cholesterol; CVD, cardiovascular disease.

Note: Date are presented as mean \pm standard deviation, or $\mathrm{n}(\%)$, as appropriate

2. Baseline $\mathrm{WH} \mathrm{R}$ in relationship to the CVD incidence

The Kaplan-Meier survival curves for the four groups according to the values of WHtR were showed in Fig. 1. The curves showed that the cumulative CVD incidence in the group with $W H t R>0.60$ was highest among four groups and was significantly higher than that in the group with $0.4 \leq \mathrm{WHtR}<0.5$. ( $\mathrm{p}$ for Logrank test $=0.003, p<0.05$ )

The association between baseline WHtR and the CVD incidence was further evaluated through multivariate Cox proportional hazards models (Table 2). Univariate analysis showed that the HR of CVD for participants with for a 1-SD Increase in WHtR by 1.21-fold $(p=0.001)$. After adjustment for age, sex, current smoking, current drinking, TC, HDL-C, LDL-C, triglyceride, diabetes, SBP and DBP, the HR (95\% Cl) for a 1-SD increase in WHtR was 1.16 (95\% Cl: 1.03-1.31) for CVD. 
Table 2

Hazard Ratios and 95\% Confidence Intervals of CVD for a 1-SD Increase in Anthropometric Indicator.

\begin{tabular}{|c|c|c|c|c|}
\hline All participants & $\begin{array}{l}\text { Unadjusted HR } \\
(95 \% \mathrm{Cl})\end{array}$ & $\begin{array}{l}P \\
\text { value }\end{array}$ & Adjusted HR (95\%Cl) & $P$ value \\
\hline \multicolumn{5}{|c|}{$\begin{array}{l}\text { Continuous (Per SD } \\
\text { increase) }\end{array}$} \\
\hline WHtR & $1.21(1.08-1.35)$ & 0.001 & $1.16(1.03-1.31)$ & 0.017 \\
\hline BMI & $1.06(0.95-1.19)$ & 0.298 & $1.15(1.03-1.29)$ & 0.016 \\
\hline WC & $1.16(1.03-1.29)$ & 0.013 & $1.13(0.99-1.28)$ & 0.053 \\
\hline WHR & $1.20(1.10-1.30)$ & $<0.001$ & $1.15(1.04-1.27)$ & 0.005 \\
\hline
\end{tabular}

Table 3 shows the HRs for CVD according to the levels of four abdominal adiposity indices. Univariated HR of CVD for participants with WHtR 0.60 increased by 1.98 -fold $(P<0.001)$, compared with the reference group. After adjustment for age, sex, current smoking, current drinking, TC, HDL-C, LDL-C, triglyceride, diabetes, SBP and DBP, the $\mathrm{HR}(95 \% \mathrm{Cl})$ for participants with WHtR 0.60 increased was 1.87 (95\% Cl: 1.23-2.83) for CVD. ( $P=0.003)$. Moreover, the participants with $0.50 \leq \mathrm{WHtR}<0.60$ still had a significant difference comparing with the reference group. The multivariate-adjusted $\mathrm{HR}(95 \% \mathrm{Cl})$ for 0.50 $\leq \mathrm{WHtR}<0.60$ was 1.45 (95\% Cl: 1.09-1.94) for CVD. However, the other three abdominal adiposity indices didn't show the such significant results. 
Table 3

Associations between risks of adverse outcomes and different values of WHtR, BMI, WC and WHR.

\begin{tabular}{|c|c|c|c|c|}
\hline All participants & Unadjusted HR (95\% Cl) & $P$ value & Adjusted HR (95\%Cl) & $P$ value \\
\hline \multicolumn{5}{|l|}{ WHtR } \\
\hline $\mathrm{WHtR}<0.4$ & $0.83(0.20-3.37)$ & 0.790 & $0.98(0.24-3.99)$ & 0.973 \\
\hline $0.4 \leq \mathrm{WHtR}<0.5$ & 1 & - & 1 & - \\
\hline $0.5 \leq \mathrm{WHtR}<0.6$ & $1.42(1.08-1.87)$ & 0.013 & $1.45(1.09-1.94)$ & 0.012 \\
\hline $\mathrm{WHtR}>0.6$ & $1.98(1.35-2.89)$ & $<0.001$ & $1.87(1.23-2.83)$ & 0.003 \\
\hline \multicolumn{5}{|l|}{ BMI } \\
\hline $\mathrm{BMI}<18.5$ & $1.42(0.33-2.40)$ & 0.496 & $1.03(0.38-2.82)$ & 0.954 \\
\hline $18.5 \leq \mathrm{BMI}<24$ & 1 & - & 1 & - \\
\hline $24 \leq \mathrm{BMI}<30$ & $1.07(0.83-1.38)$ & 0.601 & $1.12(0.86-1.47)$ & 0.399 \\
\hline $\mathrm{BMI}>30$ & $1.42(0.96-2.10)$ & 0.083 & $1.52(0.99-2.33)$ & 0.055 \\
\hline \multicolumn{5}{|l|}{ WC } \\
\hline 1st quartile & 1 & - & 1 & - \\
\hline 2nd quartile & $1.38(0.98-1.95)$ & 0.063 & $1.36(0.96-1.92)$ & 0.081 \\
\hline 3rd quartile & $1.34(0.96-1.88)$ & 0.086 & $1.28(0.90-1.81)$ & 0.170 \\
\hline 4th quartile & $1.45(1.03-2.05)$ & 0.033 & $1.33(0.92-1.92)$ & 0.133 \\
\hline \multicolumn{5}{|l|}{ WHR } \\
\hline 1st quartile & 1 & - & 1 & - \\
\hline 2nd quartile & $1.58(1.12-2.23)$ & 0.009 & $1.47(1.04-2.08)$ & 0.031 \\
\hline 3rd quartile & $1.62(1.13-2.32)$ & 0.009 & $1.43(0.99-2.07)$ & 0.059 \\
\hline 4th quartile & $1.85(1.31-2.62)$ & $<0.001$ & $1.41(0.98-2.03)$ & 0.064 \\
\hline
\end{tabular}

3. Predictive value of $\mathrm{WHtR}$ on the CVD risk against other common anthropometric indices of obesity Furthermore, we evaluated whether adding WHtR to the conventional model could improve prediction perfor-mance. Fortunately, the IDI value and NRI value suggested that the model after addition of WHtR led to a significant improvement in predicting incident stroke or CHD within 4 years (Table 4). 
Table 4

Reclassification and discrimination statistics for adverse outcomes within 4 years by WHtR,BMI, WC and WHR.

\begin{tabular}{|llll|}
\hline & NRI $(95 \%$ Cl) & IDI & P \\
\hline WHtR & $0.05(-0.01-0.12)$ & 0.0026 & 0.01 \\
\hline BMI & $-0.01(-0.05-0.03)$ & 0.0009 & 0.09 \\
\hline WC & $-0.01(-0.05-0.02)$ & 0.0007 & 0.14 \\
\hline WHR & $-0.01(-0.05-0.04)$ & 0.0013 & 0.04 \\
\hline $\begin{array}{l}\text { Conventional model: age, sex, current smoking, current drinking, TC, HDL-C, LDL-C ,triglyceride, } \\
\text { diabetes, SBP and DBP. }\end{array}$ & \\
\hline \multicolumn{2}{|l|}{ Supplemental figure S1. Study flow chart. } \\
\hline
\end{tabular}

The result showed that the NRI value of $\mathrm{WHtR}$ was 0.05 (more than 0 indicating improvement). The IDI value of WHtR was $0.0026(p=0.01)$. Based on these models, WHtR exhibited better predictive value for the CVD incidence as revealed through the IDI value and NRI value (the higher the better), than other common anthropometric indices. Similarly, baseline WHtR was also a better predictor of the CVD than BMI, WHR and WC.

\section{Discussion}

In this prospective cohort study, WHtR was associated with risk of the CVD in people with hypertension. Notably, this positive association remained significant even after adjusting for various CVD risk factors. Moreover, in the performed comparisons of the predictive value of WHtR on the CVD incidence, WHtR was better than other common anthropometric indices of obesity (BMI, WHR and WC). WHtR exhibited better predictive value for the CVD incidence than the others.

CVD is a public health challenge. The number and mortality of CVD patients is on the rise. The incidence rate of CVD was higher in rural areas than the national average level in China. Therefore, the results of our study will have important clinical predictive significance. Especially the rural population, their income is generally low, WHtR without cost is the simplest and effective method in clinical practice.

WHtR, as a rapid screening tool, can help to predict CVD risk, and then reduce the CVD incidence. Our results also indicated that addition of WHtR could improve the conventional model for predicting adverse outcomes within 4 years. In addition, our results appear to be stable because the value of WHtR for predicting adverse events remained constant. It is the best indicator of obesity that predicts CVD risk in the population with hypertension.

Recently, there are some studies that reveal the relationship between WHtR and CVD [26-28]. A study by Gelber showed that WHtR had the strongest association with CVD in men [29]. Moreover, the superiority 
of WHtR for detecting CVD risk has been reported among children and adolescents [30]. Therefore, WHtR may be considered as a good screening tool for CVD. Some studies demonstrated that WHtR was better for predicting CVD risk [31-33]. However, the result is from mostly cross-sectional studies, and the study population is not hypertensive.

The strength of current study is that it is a large prospective cohort study. It is the first study to examine the association between WHtR and CVD in hypertensive people. Moreover, confounding factors were adequately adjusted and the results were still stable. Nevertheless, our study also has limitations. Our study is a Chinese cohort, which limits the generalizability of our findings to other ethnic groups.

\section{Conclusion}

The present study emerges evidence suggesting that WHtR may constitute a simple and accurate prognostic marker of CVD risk as compared to other obesity-related indices in hypertensive people. Indeed, the present findings offer new prospective data suggesting that WHtR exhibits a positive association with the CVD incidence in Asiatic adults from the hypertensive population without preexisting CVD. WHtR appears to be a better predictor of CVD risk than the other anthropometric indices of total and central obesity. Future studies are still required to further evaluate the association between WHtR and CVD in different ethnic and patient populations.

\section{List Of Abbreviations}

WHtR, waist-to-height ratio

$\mathrm{BMI}$, body mass index

WHR, waist-to-hip

WC, waist circumference

CVD, cardiovascular disease

NCRCHS, Northeast China Rural Cardiovascular Health Study

TC, total cholesterol

$\mathrm{CHD}$, coronary heart disease

$\mathrm{NRI}$, net reclassification improvement

IDI, integrated discrimination improvement

\section{Declarations}




\section{Ethics approval and consent to participate}

The study was approved by the Ethics Committee of China Medical University (Shenyang, China) (2018194). Written informed consent was obtained from all participants.

\section{Consent for publication}

All authors have reviewed and consented to publication of the paper.

\section{Availability of Data and Materials}

The datasets supporting the conclusions of this article are included within the article.

\section{Competing interests}

None declared.

\section{Funding}

This work was supported by the National Key Research and Development Program of China (Grant \#2017YFC1307600), the Science and Technology Program of Liaoning Province, China (Grant \#2020JH1/10300002, 2019-ZD-0335) and the Program of Health Commission of Shenyang (201904).

\section{Author's contribution}

ZS participated in wrote the manuscript. DZ conducted statistical analyses. XFG and GZS assisted with critical revision of manuscript for intellectual content. LZ, ZY, YHM, YSS coordinated data collection and subjects' follow-up. FX and ZS was responsible for the study concept and design. All authors have read and approved the final manuscript.

\section{Acknowledgements}

We would like to thank Professor Liqiang Zheng for his help with data collection and data management.

\section{References}

1. Clauss SB, de Ferranti SD. Update on Preventive Cardiology. Pediatr Clin North Am. 2020;67(5):92344.

2. Moran $\mathrm{AE}$, Tzong $\mathrm{KY}$, Forouzanfar $\mathrm{MH}$, et al. Variations in ischemic heart disease burden by age, country, and income: the Global Burden of Diseases, Injuries, and Risk Factors 2010 study. Glob Heart. 2014;9(1):91-9.

3. Pekka P, Tiina L, Vesa K, Erkki V. Contribution of the North Karelia Project to International Work in CVD and NCD Prevention and Health Promotion. Glob Heart. 2016;11(2):243-6. 
4. Chen W, Gao R, Liu L, et al. Summary of Report on Chinese Cardiovascular Diseases 2017. Chin Circ J. 2018;33:1-8.

5. Shilpa NB, Frank BH. Epidemiology of obesity and diabetes and their cardi-ovascular complications. Circ Res 2016; 27;118(11):1723-1735.

6. Samuel K, David BA, Steven BH, et al. Waist circumference and cardiometabolic risk: a consensus statement from shaping America's health: association for weight management and obesity prevention; NAASO, the obesity society; the American society for nutrition; and the American diabetes association. Obesity. 2007;15(5):1061-7.

7. Hou XH, Lu JM, Weng JP, et al. Impact of waist circumference and body mass index on risk of cardiometabolic disorder and cardiovascular disease in Chinese adults: a national diabetes and metabolic disorders survey. PLoS One. 2013;8(3):e57319.

8. Karl Z, Jakob L, Margit H, et al. Body fat distribution and risk of incident ischemic stroke in men and women aged 50 to 74 years from the general population. The KORA Augsburg cohort study. PLoS One. 2018;5(2):e0191630. 13(.

9. Yukako T, Makoto W, Yoshihiro K, et al. Effect of age on the association between waist-to-height ratio and incidence of cardiovascular disease: the Suita study. J Epidemiol. 2013;5(5):351-9. 23(.

10. Margaret A, Shiun DH. Six reasons why the waist-to-height ratio is a rapid and effective global indicator for health risks of obesity and how its use could simplify the international public health message on obesity. Int J Food Sci Nutr. 2005;56(5):303-7.

11. Paul AJ, Suzanne O, Barry LC, et al. 2014 Evidence-based guideline for the management of high blood pressure in adults. JAMA. 2014;5(5):507-20. 311(.

12. Kearney PM, Whelton M, Reynolds K, Muntner P, Whelton PK, He J. Global burden of hypertension: analysis of worldwide data. Lancet. 2005;365:217-23.

13. Calhoun DA, Booth JN, Oparil S, et al. Refractory hypertension: determination of prevalence, risk factors, and comorbidities in a large, population-based cohort. Hypertension. 2014;63:451-8.

14. Alejandro TG, Iván JNG, Hynek R, José AGD. Harish Ramakrishna Management of Arterial Hypertension: 2018 ACC/AHA Versus ESC Guidelines and Perioperative Implications. J Cardiothorac Vasc Anesth. 2019;33(12):3496-503.

15. Liu XH, Bai GN, Li H, Li SP. Applying. SF-6D to measure health state utilities among the middle and old aged patients with hypertension in China. Health Qual Life Outcomes. 2020;11(1):385. 18(.

16. Li Z, Guo XF, Zheng LQ, Yang HM, Sun YX. Grim status of hypertension in rural China: results from Northeast China Rural Cardiovascular Health Study 2013. J Am Soc Hypertens. 2015;9:358-64.

17. Sun GZ, Ye N, Chen YT, Zhou Y, Li Z, Sun YX. Early repolarization pattern in the general population: prevalence and associated factors. Int J Cardiol. 2017;230:614-8.

18. Ashwell M, Gibson S. Waist-to-height ratio as an indicator of 'early health risk': simpler and more predictive than using a 'matrix' based on BMI and waist circumference. BMJ Open. 2016;14(3):e010159. 6(. 
19. Ashwell M. Shape: the waist-to-height ratio is a good, simple screening tool for cardiometabolic risk. Nutr Today. 2011;46:85-9.

20. Xu J, Xu T, Bu XQ, et al. The predictive value of waist-to-height ratio for ischemic stroke in a population-based prospective cohort study among Mongolian men in China. PLoS One. 2014;29(10):e110245. 9 (.

21. Chobanian AV, Bakris GL, Black HR, et al. The seventh report of the Joint National Committee on prevention, detection, evaluation, and treatment of high blood pres-sure: the JNC 7 report. JAMA. 2003;289:2560-72.

22. Jutta BK. What's new in the ESC 2018 guidelines for arterial hypertension: The ten most important messages. Wien Klin Wochenschr. 2019;131(7):180-5.

23. Chamberlain JJ, Rhinehart AS, Shaefer CF, Neuman A. Diagnosis and management of diabetes: synopsis of the 2016 American Diabetes Association Standards of Medical Care in Diabetes. Ann Intern Med. 2016;164:542-52.

24. Zhao D, Liu J, Wang W, et al. Epidemiological transition of stroke in China: twenty-one-year observational study from the Sino-MONICA-Beijing Project. Stroke. 2008;39:1668-74.

25. Gaye B, Canonico M, Perier MC, et al. Ideal cardiovascular health, mortality, and vascular events in elderly subjects: the three-city study. J Am Coll Cardiol. 2017;69:3015-26.

26. Karl Z, Jakob L, Margit $H$, et al. Body fat distribution and risk of incident ischemic stroke in men and women aged 50 to 74 years from the general population. The KORA Augsburg cohort study. PLoS One. 2018;5(2):e0191630. 13(.

27. Ware LG, Rennie KL, Kruger HS, et al. Evaluation of waist-to-height ratio to predict 5 year cardiometabolic risk in sub-Saharan African adults. Nutr Metab Cardiovasc Dis. 2014;24(8):900-7.

28. Ioannis K, Demosthenes BP, Georgia MK, et al. Lipid accumulation product in relation to 10-year cardiovascular disease incidence in Caucasian adults: The ATTICA study. Atherosclerosis. 2018;279:10-6.

29. Gelber RP, Gaziano JM, Orav EJ, et al. Measures of obesity and cardiovascular risk among men and women. J Am Coll Cardiol. 2008;52:605-15.

30. Savva SC, Tornaritis M, Savva ME, et al. Waist circumference and waist-to-height ratio are better predictors of cardiovascular disease risk factors in children than body mass index. Int J Obes Relat Metab Disord. 2000;24:1453-8.

31. Ashwell M, Gunn P, Gibson S. Waist-to-height ratio is a better screening tool than waist circumference and BMI for adult cardiometabolic risk factors: systematic review and meta-analysis. Obes Rev. 2012;13(3):275-86.

32. Lee CM, Huxley RR, Wildman RP, Woodward M. Indices of abdominal obesity are better discriminators of cardiovascular risk factors than BMI: a meta-analysis. J Clin Epidemiol. 2008;61(7):646e53.

33. Browning LM, Hsieh SD, Ashwell M. A systematic review of waistto-height ratio as a screening tool for the prediction of cardiovascular disease and diabetes: 0.5 could be a suitable global boundary 
value. Nutr Res Rev. 2010;23(2):247e69.

Figures

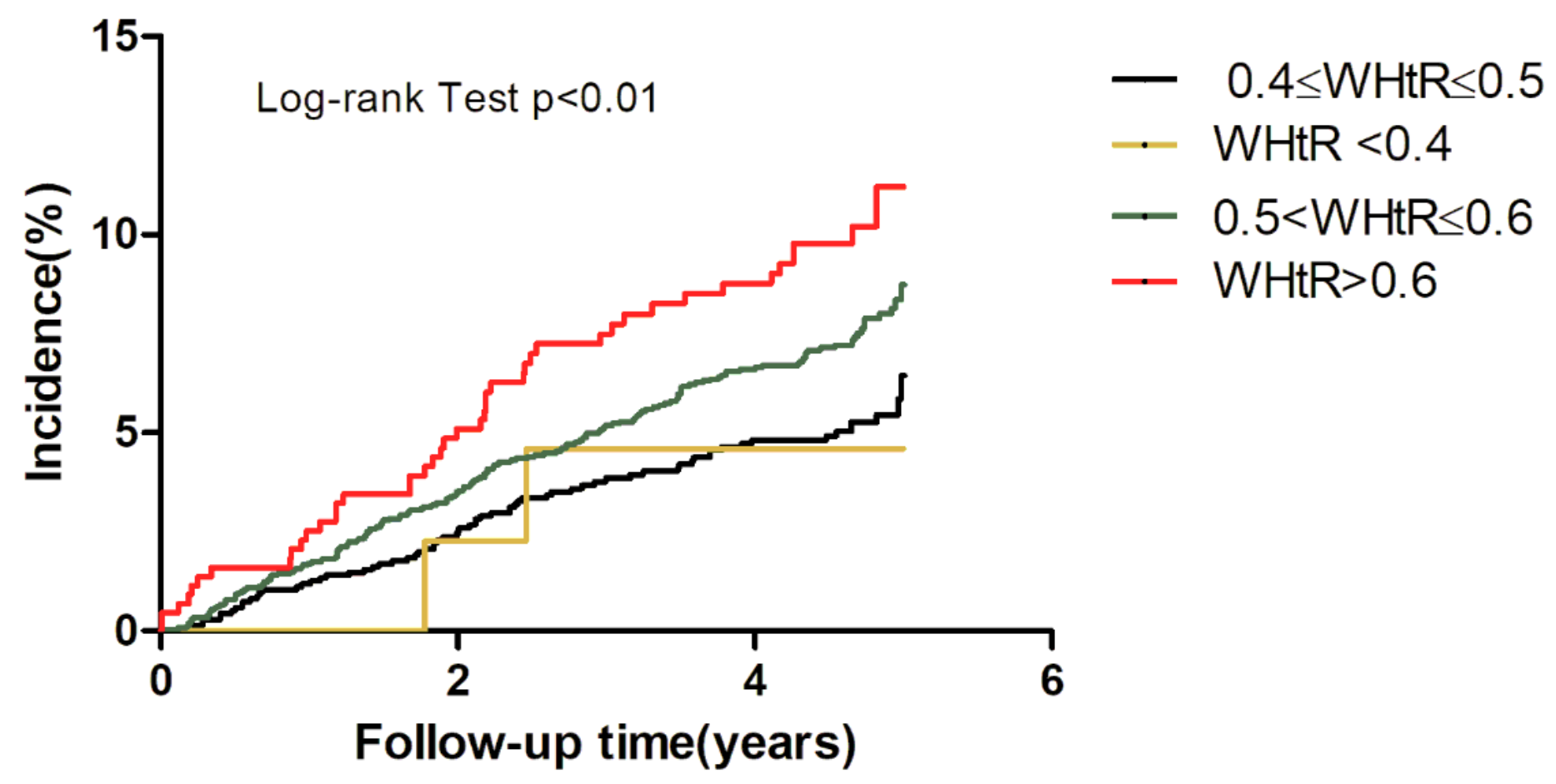

Figure 1

Unadjusted Kaplan-Meier curves for incident adverse events stratified by WHtR.

\section{Supplementary Files}

This is a list of supplementary files associated with this preprint. Click to download.

- supplemental1.docx 